\title{
Scaling Dynamical Correlation Energy from Density Functional Theory Correlation Functionals $^{\dagger}$
}

\author{
B. Ramachandran* \\ Chemistry, College of Engineering and Science, Louisiana Tech University, Ruston, Louisiana 71272
}

Received: February 2, 2005; In Final Form: April 18, 2005

\begin{abstract}
The scaling of dynamical correlation energy in molecules obtained by the correlation functionals of density functional theory (DFT) is examined. The approach taken is very similar to the scaled external correlation method of Brown and Truhlar but is based on the observation that DFT correlation functionals, especially the LYP, appear to represent the dynamical portion of the correlation energy in molecules. We examine whether higher accuracy in atomization energies can be gained by scaling without significant deterioration of the structural and spectroscopic properties of the molecules using four DFT functionals (BLYP, OLYP, B3LYP, and O3LYP) on 19 molecules including the six molecule AE6 database, the latter being representative of a much larger, 109 molecule training set. We show that, with molecule specific scale factors, nearly perfect agreement with experiment can be achieved in atomization energies without increasing the average errors in other molecular properties relative to the DFT calculation. We further show that it is possible to find optimal scale factors which reduce the mean unsigned error per bond to levels comparable to those of some multilevel multicoefficient methods.
\end{abstract}

\section{Introduction}

In this paper, we explore whether it is possible to obtain accurate atomization energies for molecules by scaling the electron correlation energy calculated by density functional theory (DFT) ${ }^{1}$ correlation functionals, without significant deterioration of the structural and spectroscopic properties of the molecules. The commonly accepted definition of the electron correlation energy of a molecule, due to Löwdin, ${ }^{2}$ is

$$
E_{\mathrm{corr}}=E-E_{\mathrm{HF}}
$$

where $E$ is the exact (nonrelativistic) energy and $E_{\mathrm{HF}}$ is the Hartree-Fock limit energy. It has been recognized for quite some time ${ }^{3}$ that $E_{\text {corr }}$ may be viewed as a composite quantity made up of at least two components: the "dynamical" or "external" correlation energy and the "nondynamical" or "internal" correlation energy. The former is attributed to the reduction in the value of the wave function as two electrons approach each other (due to the $1 / r_{i j}$ terms in the Hamiltonian) while the latter is associated with the energy lowering resulting from the interaction of the ground state Hartree-Fock configuration with low-lying excited states and has been called static, left-right, or near-degeneracy correlation energy. It is also generally accepted that dynamical correlation is only weakly dependent on relative nuclear positions, i.e., molecular geometry, while the same cannot be said for the nondynamical component because of its origins in the interactions between neighboring electronic states (the near-degeneracy aspect).

Despite this understanding of the nature of $E_{\text {corr }}$, however, it is impossible to separately calculate the two types of correlation energy in a theoretically rigorous fashion. The combination of the complete active space (CAS) self-consistent field method ${ }^{4}$

* To whom correspondence should be addressed. E-mail: ramu@latech.edu.

† Part of the special issue "Donald G. Truhlar Festschrift". with configuration interaction $(\mathrm{CI})^{5}$ comes closest to affording this separation in practice. With a "sufficiently large" basis set, the CAS method is expected to recover most of the nondynamical correlation while the CI calculation using the CAS solutions as references recovers as much of the remaining (presumably mostly dynamical) correlation energy as possible, given the twin limitations of finite one-electron basis set size and the truncation of the slow-converging CI series typically at single and double excitations (MR-CISD). The incomplete recovery of dynamical correlation by MR-CISD typically results in bond dissociation energies that are too low and reaction barrier heights that are too high.

The scaled external correlation (SEC) method of Brown and Truhlar $^{6}$ represents an attempt to overcome the limitations of the CISD method by scaling the external correlation energy, which is proportional to the difference between the CAS and the CISD energies for a given molecular geometry. The weak geometry dependence of dynamical correlation suggests a particularly simple scaling, namely, multiplication by a constant factor. So, for a diatomic molecule AB, the SEC-scaled energy is obtained as ${ }^{6}$

$$
E_{\mathrm{AB}}^{\mathrm{SEC}}=E_{\mathrm{AB}}^{\mathrm{CAS}}+\frac{1}{F_{\mathrm{AB}}}\left(E_{\mathrm{AB}}^{\mathrm{CISD}}-E_{\mathrm{AB}}^{\mathrm{CAS}}\right)
$$

where the scale factor $F_{\mathrm{AB}}$ is obtained from the BornOppenheimer bond dissociation energies, $D_{\mathrm{e}}$, as

$$
F_{\mathrm{AB}}=\frac{D_{\mathrm{e}, \mathrm{AB}}^{\mathrm{CISD}}-D_{\mathrm{e}, \mathrm{AB}}^{\mathrm{CAS}}}{D_{\mathrm{e}, \mathrm{AB}}^{\mathrm{expt}}-D_{\mathrm{e}, \mathrm{AB}}^{\mathrm{CAS}}}
$$

This method has been successfully applied to the construction of potential energy surfaces for $\mathrm{F}+\mathrm{H}_{2},{ }^{7-9} \mathrm{Cl}+\mathrm{H}_{2},{ }^{10}$ and $\mathrm{O}\left({ }^{3} \mathrm{P}\right)$ $+\mathrm{HCl}^{11-13}$ reactions as well as an accurate near-equilibrium potential energy surface for the $\mathrm{HOCl}$ molecule. ${ }^{14}$ 
Despite its simplicity and success, the applicability of the SEC method is limited to small molecules because of the computational effort involved in the CAS/CISD combination. However, it has become clear from recent investigations ${ }^{15,16}$ that the generalized gradient approximation (GGA) exchangecorrelation functionals used in DFT also provide the means of separating dynamical and nondynamical correlation energies but at considerably less computational cost. Gritsenko et al. ${ }^{16}$ note that "the GGA exchange functionals represent effectively not only exchange, but also the molecular nondynamical correlation, while the GGA correlation functionals represent dynamical correlation only (emphasis added)." By comparing the correlation energy contributions given by LYP, ${ }^{17} \mathrm{P} 86,{ }^{18}$ and $\mathrm{VWN}^{19}$ functionals for several diatomic molecules, Mok et al. ${ }^{15}$ have shown that the LYP functional appears to most closely represent the dynamical correlation and that the LYP contribution to the total energy is reasonably independent of the bond length.

These findings not only indicate that dynamical correlation for larger molecular systems can be estimated quite economically using DFT methods but also suggest the possibility that it may be possible to significantly increase the accuracy of the calculated Born-Oppenheimer equilibrium energies relative to those of the atoms by a simple scaling of the dynamical correlation. The main goal of this paper is to explore whether this can be achieved without significant deterioration of the structural and spectroscopic properties of the molecules. To distinguish our DFT-based approach from the CAS/CISD-based SEC method, we shall refer to the scaled DFT energies as the scaled dynamical correlation (SDC) energies.

The scaling of correlation energy in order to increase the accuracy of quantum chemical calculations has a long and productive history, starting with the SEC method. ${ }^{6}$ The SEC method was soon followed by the scaling all correlation (SAC) method of Gordon and Truhlar, ${ }^{20-22}$ which is based on scaling the total correlation energy, obtained as the difference between the Hartree-Fock and a single-reference correlated method (MP2 or MP4, for example). The SEC and SAC methods can be considered the forerunners of the PCI-X methods of Siegbahn and co-workers, ${ }^{23}$ the multilevel multicoefficient (MC) methods of the Truhlar group, ${ }^{24-30}$ and the G3S model chemistry of Pople and co-workers. ${ }^{31}$ More recently, the Truhlar group has developed several parametrized DFT functionals as well as MC methods that mix ab initio and DFT methods, ${ }^{32-34}$ which have proved capable of delivering "chemical accuracy" (mean absolute error less than $1 \mathrm{kcal} / \mathrm{mol}$ per bond) at less computational cost than previous methods. Some of these methods formally scale as $N^{5}$, where $N$ is the number of atoms in the molecule, as compared to the $N^{7}$ scaling of the Gaussian model chemistries and some MC methods. In contrast to these methods, the approach proposed here is characterized by its simplicity and ease of implementation and formal scaling of $N^{4}$.

The remainder of this paper is organized as follows. In section II, we describe our calculations and the conventions used for defining the scale factors. In section III, we present and discuss the results. Section IV is a summary highlighting the important conclusions.

\section{Calculations}

Molecules, Basis Sets, and DFT Functionals. We limit the present investigation to 19 molecules, six of which constitute the AE6 database of Lynch and Truhlar, ${ }^{35}$ which is representative of a 109 molecule database of atomization energies called Database/3. ${ }^{36}$ The AE6 molecules are $\mathrm{SiH}_{4}, \mathrm{SiO}, \mathrm{S}_{2}$, propyne, glyoxal, and cyclobutane. The other molecules are the diatomics
$\mathrm{HF}, \mathrm{OH}, \mathrm{OF}, \mathrm{F}_{2}, \mathrm{HCl}, \mathrm{ClO}, \mathrm{CO}, \mathrm{NH}$, and $\mathrm{NO}$, and the triatomics $\mathrm{HOF}, \mathrm{HOCl}, \mathrm{F}_{2} \mathrm{O}$, and $\mathrm{HNO}$. Accurate experimental estimations of the relevant properties for these molecules are available. Also, calculations at high levels of ab initio theory on several of them have been recently carried out by this research group. ${ }^{11,13,14,37,38}$ We use the cc-pVTZ basis set of Dunning and co-workers ${ }^{39}$ as a reasonably small, yet reasonably effective, basis set that can be used for studies of larger molecular systems.

The present study makes use of the popular exchange functional of Becke, B88 (abbreviated as B hereafter), ${ }^{40}$ the hybrid exchange functional $\mathrm{B} 3,{ }^{41}$ the OPTX functional of Handy and Cohen (abbreviated as $\mathrm{O}$ hereafter), ${ }^{42}$ and its hybrid version O3. ${ }^{43}$ These are used with the LYP correlation functional ${ }^{17}$ to yield the exchange-correlation functionals BLYP, B3LYP, OLYP, and O3LYP. All calculations are performed with the Gaussian 03 program $^{44}$ using a pruned grid of 99590 points (the "ultrafine" grid) for integral evaluations. For consistency, the exchange functionals in each case were "generated" from the exchange-correlation functional by setting the correlation part of the functional to zero using program options ${ }^{45}$ even in the case of the B88 where the stand-alone exchange functional is available in the program.

There are a few subtleties involved in the LYP correlation functionals used in these methods. The LYP correlation functional used in BLYP is an equal mixture of the local (functional III of Vosko, Wilk, and Nusair, VWN) ${ }^{46}$ and nonlocal correlation functionals, as $E^{\mathrm{LYP}}=E_{\mathrm{c}}^{\mathrm{VWN}}+\Delta E_{\mathrm{c}}^{\text {nonlocal }}$. In the formulation of $\mathrm{B} 3 \mathrm{LYP},{ }^{41}$ the correlation part is calculated as $E^{\mathrm{LYP}}=E_{\mathrm{c}}^{\mathrm{VWN}}+C \Delta E_{\mathrm{c}}^{\text {nonlocal }}$, where $C=0.810$. The LYP used in OLYP and O3LYP makes use of functional V of ref 46 (VWN5).

Dynamical Correlation Energy. For the remainder of our discussion, we will always refer to the total energies of molecules relative to those of the atoms at infinite separation. The Born-Oppenheimer atomization energy of a diatomic is then given by $D_{\mathrm{e}}=-E\left(r_{\mathrm{e}}\right)$, where $r_{\mathrm{e}}$ is the equilibrium bond distance.

The basic premise of our approach is that the difference between the energies calculated by a given exchange functional $\mathrm{M}$ (B, B3, O, and O3, in this work) and that obtained by $\mathrm{M}+$ LYP (or MLYP) for a molecule is an approximate measure of the dynamical correlation energy, i.e.,

$$
\Delta E_{\mathrm{dc}}^{\mathrm{M}}(r) \propto E_{\mathrm{MLYP}}(r)-E_{\mathrm{M}}(r)
$$

The left-hand side is labeled with $M$ because the actual energy difference depends on the density used in the LYP calculation, which depends on the choice of the exchange functional. It is now a simple matter to define the scale factor in a manner similar to the SEC method, as given in eq 3 for a diatomic molecule. However, we make a small (but for all practical purposes, insignificant) modification in this work. Because the equilibrium geometries given by M and MLYP are typically different, the $D_{\mathrm{e}}$ values at the two levels of treatment actually refer to energies at different values of the bond length. Therefore, we choose to define the scale factor with respect to the equilibrium geometry of the MLYP calculation. For example, the SDC scale factor for a diatomic, $f_{2}$, is defined as

$$
f_{2}^{\mathrm{M}}=\frac{D_{\mathrm{e}, \mathrm{expt}}+E_{\mathrm{M}}\left(r_{\mathrm{e}}^{\mathrm{MLYP}}\right)}{D_{\mathrm{e}, \mathrm{MLYP}}+E_{\mathrm{M}}\left(r_{\mathrm{e}}^{\mathrm{MLYP}}\right)}
$$

where it should be noted that the $D_{\mathrm{e}}$ values are positive numbers while the $E_{\mathrm{M}}$ values are negative. Note also that in eq 5 we 
have inverted the form of the SEC scale factor in eq 3 for purely aesthetic reasons. From a computational point of view, using only the equilibrium geometry at the MLYP level for defining the scale factor actually simplifies matters. To implement eq 5, it is sufficient to do a geometry optimization at the MLYP level followed by a single point calculation without the correlation functional (i.e., because $D_{\mathrm{e}, \mathrm{M}}$ is not required, a geometry optimization at the $\mathrm{M}$ level of treatment need not be done). Now the SDC energies of the diatomic molecule can be obtained at any interatomic distance $r$ as

$$
E_{\mathrm{M}}^{\mathrm{SDC}}(r)=E_{\mathrm{M}}(r)+f_{2}^{\mathrm{M}} \Delta E_{\mathrm{dc}}^{\mathrm{M}}(r)
$$

The scaling factors for larger molecules are also defined in a similar manner, using the MLYP equilibrium geometry as the reference. For example, for a triatomic molecule, we define

$$
f_{3}^{\mathrm{M}}=\frac{D_{\mathrm{e}, \text { expt }}+E_{\mathrm{M}}\left(r_{1}^{\mathrm{e}}, r_{2}^{\mathrm{e}}, r_{3}^{\mathrm{e}}\right)}{D_{\mathrm{e}, \mathrm{MLYP}}+E_{\mathrm{M}}\left(r_{1}^{\mathrm{e}}, r_{2}^{\mathrm{e}}, r_{3}^{\mathrm{e}}\right)}
$$

where $D_{\mathrm{e}}$ refers to the atomization energy and $\left(r_{1}^{\mathrm{e}}, r_{2}^{\mathrm{e}}, r_{3}^{\mathrm{e}}\right)$ is the MLYP equilibrium geometry.

Exceptions to using MLYP geometries as the reference geometry for defining scale factors were made in the case of the AE6 molecules. In these cases, to facilitate comparisons with the results of the Truhlar group, ${ }^{32}$ we used single point calculations at M and MLYP levels of treatment using the QCISD/MG3 optimized geometries provided in the AE6 database. ${ }^{47}$

Potential Energy Curves and Surfaces. Equilibrium geometries and spectroscopic constants of the molecules including the anharmonic contributions are calculated from near-equilibrium potential energy curves for $\mathrm{HF}, \mathrm{OH}, \mathrm{OF}, \mathrm{F}_{2}, \mathrm{HCl}, \mathrm{ClO}$, $\mathrm{CO}, \mathrm{NH}$, and $\mathrm{NO}$ and near-equilibrium potential energy surfaces for $\mathrm{HOF}, \mathrm{HOCl}$, and $\mathrm{HNO}$. The approach used is identical to that used by us in a recent detailed study of the HOF molecule. ${ }^{38}$ The spectroscopic properties of the diatomics are calculated using a sixth-order polynomial interpolation through seven energies spanning the experimental equilibrium geometry. For a triatomic molecule $\mathrm{ABC}, 41$ points at $\mathrm{M}$ and MLYP levels are calculated in $\left(r_{\mathrm{AB}}, r_{\mathrm{BC}}, \theta_{\mathrm{ABC}}\right)$ space spanning the experimental equilibrium geometry. These energies as well as the SDC-scaled energies are then fit to polynomial expansions using the program SURFIT, ${ }^{48}$ using displacement coordinates for the bond lengths and the Carter-Handy coordinate ${ }^{49}$ for the bend. A fourth-order expansion in all three coordinates was employed along with fifth- and sixth-order "diagonal" terms in each coordinate. This results in a polynomial with 41 linear parameters so that the fit is, in effect, an interpolation essentially free of statistical error. Analysis of the properties of the resulting potential energy surfaces leads to equilibrium geometries and spectroscopic constants, which can be compared with experimental values.

\section{Results and Discussion}

SDC Scale Factors. Table 1 presents the SDC scale factors for the molecules along with the average and the standard deviation for each exchange functional M. We find scale factors $f^{\mathrm{M}}$ that are greater than unity when $D_{\mathrm{e}, \mathrm{MLYP}}<D_{\mathrm{e}, \text { expt }}$ but also cases where $f^{\mathrm{M}}$ is less than unity, where $D_{\mathrm{e}, \mathrm{MLYP}}>D_{\text {e,expt }}$. The $f^{\mathrm{B}}$ and $f^{\mathrm{O}}$ for $\mathrm{OF}, \mathrm{F}_{2}$, and $\mathrm{F}_{2} \mathrm{O}$ are significantly less than 1 indicating that the LYP correlation functional substantially overestimates the molecular dynamical correlation energy from these densities. The pure methods also have a larger scatter of
TABLE 1: SDC Scale Factors $f^{\mathrm{M}}$

\begin{tabular}{lcccc}
\hline & $\mathrm{B}$ & $\mathrm{O}$ & $\mathrm{B} 3$ & $\mathrm{O} 3$ \\
\hline $\mathrm{HF}$ & 1.108427 & 1.029718 & 1.164986 & 1.060145 \\
$\mathrm{OH}$ & 0.965258 & 1.007404 & 1.015528 & 1.018831 \\
$\mathrm{OF}$ & 0.412050 & 0.545252 & 0.889963 & 0.780723 \\
$\mathrm{~F}_{2}$ & 0.396757 & 0.526860 & 1.021846 & 0.838733 \\
$\mathrm{HCl}$ & 1.159547 & 1.021272 & 1.131417 & 1.016324 \\
$\mathrm{ClO}$ & 0.738678 & 0.796501 & 1.061436 & 0.966077 \\
$\mathrm{NH}$ & 0.823059 & 0.935334 & 0.877600 & 0.943915 \\
$\mathrm{NO}$ & 0.766996 & 0.892274 & 1.006969 & 1.014244 \\
$\mathrm{CO}$ & 0.937721 & 1.001976 & 1.101505 & 1.088297 \\
$\mathrm{HOF}$ & 0.793900 & 0.849127 & 1.019291 & 0.956728 \\
$\mathrm{HOCl}$ & 0.970175 & 0.981748 & 1.098532 & 1.044011 \\
$\mathrm{~F}_{2} \mathrm{O}$ & 0.481746 & 0.616681 & 0.982423 & 0.857539 \\
$\mathrm{HNO}$ & 0.826524 & 0.900934 & 0.995856 & 0.984842 \\
$\mathrm{SiH}$ & 1.085448 & 1.061249 & 1.009142 & 1.007343 \\
$\mathrm{SiO}$ & 1.001701 & 1.171089 & 1.196021 & 1.261147 \\
$\mathrm{~S}_{2}$ & 0.934626 & 0.873570 & 1.069214 & 0.960998 \\
propyne & 1.024358 & 1.000357 & 1.019366 & 0.993297 \\
glyoxal & 0.947666 & 0.955656 & 1.027137 & 0.995864 \\
cyclobutane & 1.073010 & 1.009573 & 1.030523 & 0.986479 \\
mean & 0.865666 & 0.904030 & 1.037829 & 0.988186 \\
$\sigma$ & 0.226715 & 0.174032 & 0.079925 & 0.100643
\end{tabular}

TABLE 2: Comparison of the MLYP Atomization Energies $D_{\mathrm{e}}$ (in kcal/mol) with Experimental Values ${ }^{a}$

\begin{tabular}{lrrrrrr}
\hline & BLYP & OLYP & B3LYP & O3LYP & B/SDC & expt $^{b}$ \\
\hline $\mathrm{HF}$ & 138.69 & 140.70 & 137.14 & 139.90 & 141.46 & 141.46 \\
$\mathrm{OH}$ & 108.10 & 106.97 & 106.74 & 106.66 & 107.17 & $107.17^{c}$ \\
$\mathrm{OF}$ & 68.53 & 65.36 & 56.16 & 58.88 & 53.49 & $53.37^{d}$ \\
$\mathrm{~F}_{2}$ & 50.58 & 48.23 & 38.05 & 41.65 & 38.59 & 38.39 \\
$\mathrm{HCl}$ & 103.74 & 106.84 & 104.30 & 106.95 & 107.32 & 107.32 \\
$\mathrm{ClO}$ & 72.37 & 70.93 & 63.61 & 66.16 & 65.29 & 65.26 \\
$\mathrm{CO}$ & 262.28 & 259.53 & 255.28 & 255.93 & 259.62 & 259.62 \\
$\mathrm{NH}$ & 88.57 & 85.46 & 87.14 & 85.24 & 83.67 & 83.67 \\
$\mathrm{NO}$ & 166.60 & 160.52 & 154.92 & 154.58 & 155.27 & 155.26 \\
$\mathrm{HOF}$ & 167.86 & 165.34 & 157.11 & 160.10 & 158.06 & $158.04^{e}$ \\
$\mathrm{HOCl}$ & 167.31 & 166.78 & 161.16 & 163.80 & 165.90 & 165.90 \\
$\mathrm{HNO}$ & 217.16 & 212.12 & 205.63 & 206.37 & 205.35 & 205.34 \\
$\mathrm{~F}_{2} \mathrm{O}$ & 116.81 & 111.05 & 94.65 & 100.07 & & 93.88 \\
$\mathrm{SiH}$ & 316.45 & 318.24 & 322.12 & 322.26 & & $322.83^{f}$ \\
$\mathrm{SiO}$ & 192.66 & 185.81 & 184.83 & 182.42 & & $192.73^{f}$ \\
$\mathrm{~S}_{2}$ & 104.77 & 106.64 & 100.75 & 103.92 & & $102.79^{f}$ \\
propyne & 701.60 & 705.01 & 702.29 & 706.01 & & $705.06^{f}$ \\
glyoxal & 640.95 & 639.89 & 630.35 & 634.50 & & $633.96^{f}$ \\
cyclobutane & 1131.56 & 1147.03 & 1141.86 & 1152.66 & & $1149.37^{f}$ \\
MUE (kcal/mol) & 7.54 & 4.83 & 2.74 & 2.43 & 0.02 & \\
max UE (kcal/mol) & 22.93 & 17.17 & 7.90 & 10.31 & 0.12 & \\
& & & & & &
\end{tabular}

${ }^{a}$ The B/SDC results are also shown for molecules for which a SDCscaled potential curve or surface was constructed. The MUE, defined as $\left|D_{\mathrm{e}, \text { expt }}-D_{\mathrm{e}, \text { calcd }}\right|$, and the maximum unsigned error are also shown (in $\mathrm{kcal} / \mathrm{mol}$ ). ${ }^{b}$ From refs 43 and 45 unless otherwise specified. ${ }^{c}$ Ref 44. ${ }^{d} \operatorname{Ref} 47 .{ }^{e} \operatorname{Ref} 31 .{ }^{f} \operatorname{Ref} 28$.

the scale factors, indicated by standard deviations, which are roughly twice as large as those for the corresponding hybrid methods. Both of the hybrid functionals yield average scale factors close to unity and have smaller standard deviations, but a few ( $\mathrm{HF}, \mathrm{HCl}$, and $\mathrm{SiO}$ for $\mathrm{B} 3$ and $\mathrm{SiO}$ for $\mathrm{O} 3$ ) are significantly greater than 1 .

Table 2 presents the Born-Oppenheimer atomization energies $D_{\mathrm{e}}$ of the molecules at the MLYP level of theory and the B/SDCscaled $D_{\mathrm{e}}$ values for the molecules for which we constructed a potential curve or surface. The scaled $D_{\mathrm{e}}$ from the other DFT functionals are not shown since they are very similar to those given in the $\mathrm{B} / \mathrm{SDC}$ column. Also given are the experimental $D_{\text {e values }}{ }^{50-55}$ which have been adjusted for spin-orbit splittings ${ }^{56,57}$ so as to facilitate fair comparisons with the nonrelativistic calculations. The mean unsigned error (MUE) for this set, defined as the average of $\left|D_{\mathrm{e}, \text { calcd }}-D_{\text {e,expt }}\right|$, and the maximum unsigned error are also shown for each method. The smaller errors for the hybrid methods are consistent with many studies that have compared the performance of DFT functionals for much larger sets of molecules. ${ }^{35,36,58-63}$ The SDC scaling in each case yields $D_{\mathrm{e}}$ values very close to the experimental 
TABLE 3: Comparison of the MLYP/cc-pVTZ and SDC-Scaled Diatomic Molecular Properties with Experiment

\begin{tabular}{|c|c|c|c|c|c|c|c|c|c|}
\hline & BLYP & OLYP & B3LYP & O3LYP & $\mathrm{B} / \mathrm{SDC}$ & $\mathrm{O} / \mathrm{SDC}$ & B3/SDC & $\mathrm{O} 3 / \mathrm{SDC}$ & expt \\
\hline \multicolumn{10}{|c|}{ equilibrium bond distances $r_{\mathrm{e}}(\AA)$} \\
\hline $\mathrm{HF}$ & 0.9330 & 0.9233 & 0.9223 & 0.9188 & 0.9321 & 0.9231 & 0.9209 & 0.9184 & 0.9168 \\
\hline $\mathrm{OH}$ & 0.9857 & 0.9784 & 0.9746 & 0.9733 & 0.9861 & 0.9783 & 0.9745 & 0.9731 & 0.9697 \\
\hline $\mathrm{OF}$ & 1.3802 & 1.3541 & 1.3503 & 1.3412 & 1.3977 & 1.3668 & 1.3533 & 1.3469 & 1.3542 \\
\hline $\mathrm{F}_{2}$ & 1.4331 & 1.4062 & 1.3976 & 1.3900 & 1.4502 & 1.4190 & 1.3971 & 1.3938 & 1.4119 \\
\hline $\mathrm{HCl}$ & 1.2931 & 1.2841 & 1.2834 & 1.2803 & 1.2911 & 1.2839 & 1.2817 & 1.2801 & 1.2746 \\
\hline $\mathrm{ClO}$ & 1.6190 & 1.5901 & 1.5934 & 1.5806 & 1.6290 & 1.5972 & 1.5912 & 1.5817 & 1.5696 \\
\hline $\mathrm{CO}$ & 1.1379 & 1.1377 & 1.1262 & 1.1307 & 1.1386 & 1.1378 & 1.1251 & 1.1298 & 1.1283 \\
\hline $\mathrm{NH}$ & 1.0520 & 1.0489 & 1.0410 & 1.0431 & 1.0543 & 1.0498 & 1.0427 & 1.0439 & 1.0362 \\
\hline NO & 1.1628 & 1.1570 & 1.1460 & 1.1482 & 1.1660 & 1.1584 & 1.1460 & 1.1480 & 1.1508 \\
\hline MUFE & 0.0165 & 0.0074 & 0.0063 & 0.0060 & 0.0204 & 0.0092 & 0.0059 & 0.0052 & \\
\hline \multicolumn{10}{|c|}{ harmonic frequencies, $\omega_{\mathrm{e}}\left(\mathrm{cm}^{-1}\right)$} \\
\hline $\mathrm{HF}$ & 3930.87 & 4052.82 & 4089.18 & 4125.39 & 3942.31 & 4055.93 & 4106.97 & 4131.84 & 4138.33 \\
\hline $\mathrm{OH}$ & 3547.58 & 3639.15 & 3697.11 & 3710.94 & 3543.47 & 3640.03 & 3699.00 & 3713.24 & 3737.76 \\
\hline $\mathrm{OF}$ & 1045.78 & 1101.39 & 1118.09 & 1137.13 & 1002.98 & 1066.24 & 1109.80 & 1120.55 & 1053.01 \\
\hline $\mathrm{F}_{2}$ & 963.09 & 1004.12 & 1049.68 & 1050.89 & 926.79 & 974.18 & 1050.91 & 1041.57 & 916.64 \\
\hline $\mathrm{HCl}$ & 2847.12 & 2930.37 & 2940.91 & 2969.71 & 2861.41 & 2932.23 & 2953.03 & 2971.18 & 2990.95 \\
\hline $\mathrm{ClO}$ & 803.53 & 859.09 & 846.94 & 876.55 & 785.28 & 843.55 & 851.59 & 873.96 & 853.8 \\
\hline $\mathrm{CO}$ & 2113.99 & 2129.66 & 2211.59 & 2184.64 & 2109.62 & 2129.50 & 2217.79 & 2189.91 & 2169.81 \\
\hline $\mathrm{NH}$ & 3123.55 & 3166.95 & 3250.62 & 3232.48 & 3101.42 & 3158.51 & 3234.32 & 3224.86 & 3282.2 \\
\hline NO & 1848.37 & 1904.57 & 1976.66 & 1969.36 & 1830.31 & 1896.31 & 1976.89 & 1970.10 & 1904.2 \\
\hline MUFE & 0.0410 & 0.0299 & 0.0357 & 0.0363 & 0.0448 & 0.0237 & 0.0343 & 0.0333 & \\
\hline \multicolumn{10}{|c|}{ anharmonicity constants, $\omega_{\mathrm{e}} x_{\mathrm{e}}\left(\mathrm{cm}^{-1}\right)$} \\
\hline $\mathrm{HF}$ & 86.14 & 88.54 & 84.95 & 87.45 & 86.04 & 88.52 & 84.91 & 87.43 & 89.881 \\
\hline $\mathrm{OH}$ & 80.55 & 82.69 & 79.77 & 81.89 & 80.56 & 82.69 & 79.77 & 81.89 & 84.9 \\
\hline $\mathrm{OF}$ & 6.68 & 7.41 & 7.11 & 7.59 & 6.95 & 7.62 & 7.16 & 7.70 & 9.919 \\
\hline $\mathrm{F}_{2}$ & 6.46 & 6.90 & 6.40 & 6.81 & 6.59 & 6.95 & 6.41 & 6.80 & 11.24 \\
\hline $\mathrm{HCl}$ & 44.72 & 45.77 & 44.81 & 45.80 & 44.65 & 45.77 & 44.82 & 45.81 & 52.8 \\
\hline $\mathrm{ClO}$ & 3.81 & 4.63 & 4.01 & 4.92 & 3.96 & 4.45 & 4.00 & 4.90 & 5.5 \\
\hline $\mathrm{CO}$ & 12.86 & 12.46 & 12.25 & 12.21 & 12.86 & 12.46 & 12.25 & 12.21 & 13.29 \\
\hline NH & 72.17 & 71.75 & 69.98 & 70.38 & 72.79 & 72.09 & 70.39 & 70.65 & 78.3 \\
\hline NO & 12.81 & 12.35 & 12.25 & 12.12 & 12.84 & 12.37 & 12.25 & 12.12 & 14.075 \\
\hline MUFE & 0.1672 & $\mathbf{0 . 1 3 7 7}$ & 0.1739 & 0.1390 & 0.1591 & 0.1379 & 0.1730 & 0.1379 & \\
\hline \multicolumn{10}{|c|}{ rotational-vibrational couplings, $\alpha_{\mathrm{e}}\left(\mathrm{cm}^{-1}\right)$} \\
\hline $\mathrm{HF}$ & 0.7874 & 0.7983 & 0.7733 & 0.7877 & 0.7867 & 0.7981 & 0.7729 & 0.7875 & 0.7981 \\
\hline $\mathrm{OH}$ & 0.7305 & 0.7308 & 0.7093 & 0.7182 & 0.7309 & 0.7307 & 0.7092 & 0.7180 & 0.724 \\
\hline $\mathrm{OF}$ & 0.0101 & 0.0109 & 0.0106 & 0.0110 & 0.0103 & 0.0111 & 0.0106 & 0.0111 & 0.0133 \\
\hline $\mathrm{F}_{2}$ & 0.0085 & 0.0091 & 0.0085 & 0.0090 & 0.0086 & 0.0092 & 0.0085 & 0.0090 & 0.0138 \\
\hline $\mathrm{HCl}$ & 0.3087 & 0.3085 & 0.3011 & 0.3038 & 0.3088 & 0.3085 & 0.3013 & 0.3038 & 0.307 \\
\hline $\mathrm{ClO}$ & 0.0049 & 0.0055 & 0.0052 & 0.0055 & 0.0049 & 0.0055 & 0.0052 & 0.0055 & 0.0058 \\
\hline $\mathrm{CO}$ & 0.0174 & 0.0170 & 0.0166 & 0.0166 & 0.0175 & 0.0170 & 0.0166 & 0.0166 & 0.0175 \\
\hline NH & 0.6409 & 0.6322 & 0.6212 & 0.6232 & 0.6441 & 0.6336 & 0.6233 & 0.6243 & 0.649 \\
\hline NO & 0.0170 & 0.0163 & 0.0160 & 0.0159 & 0.0171 & 0.0163 & 0.0160 & 0.0159 & 0.0171 \\
\hline MUFE & 0.0914 & 0.0767 & 0.1029 & 0.0855 & $\mathbf{0 . 0 8 9 0}$ & 0.0744 & $\mathbf{0 . 1 0 2 3}$ & 0.0848 & \\
\hline
\end{tabular}

value as indicated by the $\mathrm{B} / \mathrm{SDC}$ results shown. The MUEs for $\mathrm{O} / \mathrm{SDC}, \mathrm{B} 3 / \mathrm{SDC}$, and O3/SDC are each less than $0.01 \mathrm{kcal} /$ mol, and the maximum unsigned errors are $0.07,0.01$, and 0.02 $\mathrm{kcal} / \mathrm{mol}$, respectively. It should be noted that the SDC atomization energies for all molecules listed in the B/SDC column are calculated from the minima located on the potential curves/surfaces. The fact that these atomization energies are very close to the experimental values indicates that the minima on the scaled potential curves/surfaces lie close to the MLYP equilibrium geometry. Further confirmation of this observation is presented in the following subsection. The SDC-scaled $D_{\mathrm{e}}$ values for $\mathrm{F}_{2} \mathrm{O}$ and the AE6 molecules are not listed in Table 2 for the following reasons. For $\mathrm{F}_{2} \mathrm{O}$, the DFT energies exhibit unphysical oscillations as the bond lengths and angles are varied for all exchange functionals. Therefore, no potential energy surfaces were constructed for this molecule, and the scale factors were defined with respect to the MLYP equilibrium geometries found by geometry optimization within Gaussian 03. For the AE6 molecules, as noted above, we used the optimized geometry provided in the database, to facilitate comparisons with AE6 results using other methods. The scaling, in these cases, then guarantees that the energy difference between the reference geometries (MLYP for $\mathrm{F}_{2} \mathrm{O}$, QCISD/MG3 for AE6) and those of the atoms will exactly match the experimental value. Because including these cases in Table 1 will only serve to artificially lower the standard deviation for the B/SDC column, we have refrained from doing so.
SDC Scaling and Molecular Properties. We now examine the consequences of SDC scaling on the properties that depend on the slope and curvature of the potential surfaces, namely, the equilibrium geometries and spectroscopic properties. Table 3 presents the equilibrium geometries, the harmonic vibrational frequency, the anharmonicity constants, and the rotationvibration coupling constants for several diatomic molecules. These properties are calculated by analyzing the near-equilibrium potential energy curves at the indicated levels of calculation, as described in the third part of section II. We have not tabulated the rotational constants since they are directly related to the equilibrium geometry. We also define an unsigned fractional error so as to provide a means of comparing the accuracy of the calculated properties. For a property $P$ under consideration, we define the unsigned fractional error as

$$
\epsilon_{P}=\left|1-\frac{P^{\mathrm{calcd}}}{P^{\text {expt }}}\right|
$$

The mean unsigned fractional errors (MUFE) are also tabulated in Table 3.

It is clear from Table 3 that the MUFE for each property examined is roughly the same for the MLYP and M/SDC columns. This shows that the SDC scaling does not result in poorer agreement between the calculated and the experimental properties for the diatomics. This is an indication that the correlation energy given by the LYP functional is only weakly dependent on the bond distance, as one would expect for 
TABLE 4: Comparison of the MLYP/cc-pVTZ and SDC-Scaled Triatomic Equilibrium Geometries and Vibrational Fundamentals with Experiment

\begin{tabular}{|c|c|c|c|c|c|c|c|c|c|}
\hline & BLYP & OLYP & B3LYP & O3LYP & $\mathrm{B} / \mathrm{SDC}$ & $\mathrm{O} / \mathrm{SDC}$ & $\mathrm{B} 3 / \mathrm{SDC}$ & $\mathrm{O} 3 / \mathrm{SDC}$ & expt \\
\hline \multicolumn{10}{|c|}{ equilibrium geometry } \\
\hline $\mathrm{HOF}$ & & & & & & & & & \\
\hline $\begin{array}{l}r_{\mathrm{OH}}(\AA) \\
r_{\mathrm{OF}}(\AA)\end{array}$ & $\begin{array}{l}0.9816 \\
1.4673\end{array}$ & $\begin{array}{l}0.9739 \\
1.4373\end{array}$ & $\begin{array}{l}0.9702 \\
1.4300\end{array}$ & $\begin{array}{l}0.9687 \\
1.4207\end{array}$ & $\begin{array}{l}0.9837 \\
1.4739\end{array}$ & $\begin{array}{l}0.9754 \\
1.4418\end{array}$ & $\begin{array}{l}0.9700 \\
1.4295\end{array}$ & $\begin{array}{l}0.9691 \\
1.4219\end{array}$ & $\begin{array}{l}0.9657 \\
1.4350\end{array}$ \\
\hline $\begin{array}{l}\theta_{\mathrm{HOF}}(\mathrm{deg}) \\
\mathrm{HOCl}\end{array}$ & 97.40 & 97.95 & 98.44 & 98.46 & 97.32 & 97.90 & 98.44 & 98.45 & 97.54 \\
\hline$r_{\mathrm{OH}}(\AA)$ & 0.9780 & 0.9702 & 0.9669 & 0.9652 & 0.9783 & 0.9704 & 0.9659 & 0.9648 & 0.9644 \\
\hline$r_{\mathrm{OCl}}(\AA)$ & 1.7468 & 1.7118 & 1.7099 & 1.6973 & 1.7480 & 1.7125 & 1.7064 & 1.6958 & 1.6890 \\
\hline$\theta_{\mathrm{HOCl}}(\mathrm{deg})$ & 101.61 & 101.79 & 102.82 & 102.45 & 101.59 & 101.78 & 102.88 & 102.48 & 102.97 \\
\hline $\mathrm{HNO}$ & & & & & & & & & \\
\hline$r_{\mathrm{NH}}(\AA)$ & $\begin{array}{l}1.0810 \\
12152\end{array}$ & 1.0795 & 1.0631 & 1.0689 & 1.0839 & 1.0812 & 1.0632 & 1.0692 & 1.0903 \\
\hline$r_{\mathrm{NO}}(\AA)$ & 1.2152 & 1.2051 & 1.1983 & 1.1972 & 1.2177 & 1.2064 & 1.1984 & 1.1975 & 1.2090 \\
\hline$\theta_{\mathrm{HNO}}(\mathrm{deg})$ & 108.53 & 108.52 & 108.69 & 108.60 & 108.521 & 108.52 & 108.68 & 108.60 & 108.05 \\
\hline MUFE & 0.0133 & 0.0070 & 0.0082 & 0.0075 & 0.0142 & 0.0072 & 0.0078 & 0.0072 & \\
\hline \multicolumn{10}{|c|}{ vibrational fundamentals $\left(\mathrm{cm}^{-1}\right)$} \\
\hline $\mathrm{HOF}$ & & 9308 & 9690 & 9749 & 8721 & & & & \\
\hline $\begin{array}{l}\nu_{\mathrm{s}} \\
\nu_{\mathrm{b}}\end{array}$ & $\begin{array}{r}884.9 \\
1292.3\end{array}$ & $\begin{array}{r}930.8 \\
1338.2\end{array}$ & $\begin{array}{r}969.0 \\
1367.0\end{array}$ & $\begin{array}{r}974.9 \\
1374.1\end{array}$ & $\begin{array}{r}872.1 \\
1284.9\end{array}$ & $\begin{array}{r}921.0 \\
1333.0\end{array}$ & $\begin{array}{r}970.1 \\
1367.6\end{array}$ & $\begin{array}{r}972.4 \\
1372.7\end{array}$ & $\begin{array}{r}889.1 \\
1353.4\end{array}$ \\
\hline $\begin{array}{l}v_{\mathrm{a}} \\
\mathrm{HOCl}\end{array}$ & 3391.6 & 3484.7 & 3552.9 & 3563.5 & 3368.6 & 3468.1 & 3555.1 & 3558.7 & 3577.9 \\
\hline$v_{\mathrm{S}}$ & 668.5 & 714.5 & 727.2 & 741.9 & 666.9 & 713.5 & 732.2 & 744.2 & 724.4 \\
\hline$v_{\mathrm{b}}$ & 1187.9 & 1216.6 & 1235.4 & 1239.0 & 1187.3 & 1216.3 & 1237.2 & 1239.8 & 1238.6 \\
\hline $\begin{array}{l}v_{\mathrm{a}} \\
\mathrm{HNO}\end{array}$ & 3433.8 & 3533.5 & 3593.0 & 3609.2 & 3430.4 & 3531.4 & 3604.4 & 3614.2 & 3609.5 \\
\hline$v_{1}$ & 2349.5 & 2392.0 & 2582.5 & 2521.7 & 2321.3 & 2375.9 & 2581.4 & 2519.0 & 2683.95 \\
\hline$v_{2}$ & 1450.0 & 1477.5 & 1520.8 & 1511.9 & 1441.7 & 1473.8 & 1520.6 & 1511.3 & 1500.83 \\
\hline$v_{3}$ & 1528.7 & 1588.8 & 1638.9 & 1644.2 & 1517.8 & 1581.8 & 1638.4 & 1643.0 & 1565.34 \\
\hline MUFE & 0.0501 & 0.0307 & 0.0240 & $\mathbf{0 . 0 2 8 7}$ & 0.0559 & 0.0311 & 0.0244 & 0.0290 & \\
\hline
\end{tabular}

dynamical correlation energy. This appears to hold true even in the case of $\mathrm{OF}$ and $\mathrm{F}_{2}$, the two cases in which $f^{\mathrm{B}}$ and $f^{\mathrm{O}}$ are much less than 1.0.

Table 4 presents the comparison of MLYP and M/SDC properties for the three triatomics for which potential energy surfaces were constructed and analyzed. We have tabulated the vibrational fundamentals rather than the harmonics because the former include the effects of anharmonicity. The MUFE for the properties in Table 4 once again follows the pattern observed in Table 3 in that SDC scaling does not seriously affect the properties at the MLYP level of theory.

Optimal SDC Scale Factors. Tables 3 and 4 show that it is indeed possible to scale the LYP correlation energy of molecules with constant multiplicative scale factors without worsening the agreement between calculated and experimental structural and spectroscopic properties. However, the utility of such scaling would be far greater if it were possible to identify optimal scale factors that can be applied universally and yield atomization energies that are in better agreement with experiment than those obtained from the MLYP calculation. In this section, we explore whether this is possible, using the 19 molecules investigated here as our database.

From eq 6, we write

$$
D_{\mathrm{e}, \text { expt }}=-E_{\mathrm{M}}\left(r_{\mathrm{e}}^{\mathrm{MLYP}}\right)-f_{2}^{\mathrm{M}} \Delta E_{\mathrm{dc}}^{\mathrm{M}}\left(r_{\mathrm{e}}^{\mathrm{MLYP}}\right)
$$

with the recognition that $D_{\mathrm{e}, \operatorname{expt}} \equiv-E_{\mathrm{M}}^{\mathrm{SDC}}\left(r_{\mathrm{e}}^{\mathrm{MLYP}}\right)$. It is easy to see that a linear least squares (LS) analysis will now yield an optimal value of the scale factor. In fact, we plot $D_{\text {e,expt }}+E_{\mathrm{M}}$ as a function of $-\Delta E_{\mathrm{dc}}^{\mathrm{M}}$ and constrain the fit to pass through the origin. Figure 1 shows the results of this analysis. Table 5 presents the LS scale factors and the resulting atomization energies.

The LS scale factors obtained for all 19 molecules are shown in Table 5, part A. Comparing the MUE for this set with those given in Table 2, it appears that the scaling does not significantly change the MLYP results. The maximum errors for B/SDC and O3/SDC are, in fact, slightly higher than those for BLYP and O3LYP given in Table 2. However, as we shall see below, large errors in SDC atomization energies for a small number of molecules are responsible for the large MUE in part A of Table 5. Part B of Table 5 shows the results of LS analysis over the six AE6 molecules. The MUEs at the MLYP/cc-pVTZ level (i.e., unscaled) for the AE6 set are 6.12, 3.94, 4.09, and 2.80 $\mathrm{kcal} / \mathrm{mol}$ for BLYP, OLYP, B3LYP, and O3LYP, respectively. For B3LYP, the SDC scaling is able to achieve substantial improvement over this, yielding an MUE of $1.91 \mathrm{kcal} / \mathrm{mol}$.

It is clear from Figure 1 that further improvements can be achieved for the full set of 19 molecules by a slightly more flexible approach. As shown in Figure 1, the trend in correlation energy of the three compounds $\mathrm{OF}, \mathrm{F}_{2}$, and $\mathrm{F}_{2} \mathrm{O}$ is qualitatively different from the others, especially in the cases of $\mathrm{B}, \mathrm{O}$, and $\mathrm{O} 3$ methods. Therefore, in part $\mathrm{C}$ of Table 5 , we have considered the LS fit excluding these three molecules. The results confirm that the MUE can be lowered by excluding this set. Part D of Table 5 shows the results of an LS analysis over only the three fluorine-containing molecules excluded in part $\mathrm{C}$. The scale factors are significantly less than 1, indicating that DFT functionals overestimate the dynamical correlation energy for these molecules. In part E, we calculate the atomization energies for the full set of 19 molecules but using the $f^{\mathrm{M}}$ from part D for $\mathrm{OF}, \mathrm{F}_{2}$, and $\mathrm{F}_{2} \mathrm{O}$ and that from part $\mathrm{C}$ for the remaining molecules. The MUEs for B, O, B3, and $\mathrm{O} 3$ are now, respectively, 5.10, 3.06, 1.94, and $1.68 \mathrm{kcal} / \mathrm{mol}$. Comparing these errors with the MUEs of the MLYP energies in Table 2, it is clear that SDC scaling using LS scale factors can, in fact, reduce the mean errors in DFT atomization energies. For the pure DFT methods, part E of Table 5 also reports substantially smaller maximum errors than those in Table 2.

It is somewhat discouraging, however, that the maximum error reported for the B3/SDC atomization energies is only marginally smaller than that in Table 2 , and the maximum error reported for O3/SDC is actually slightly larger. It is possible to substantially decrease these errors by removing a single molecule from the set. This molecule is $\mathrm{SiO}$, which appears as a solid square at $-\Delta E_{\mathrm{dc}} \approx 0.06$ in Figure $1 \mathrm{c}$, d. The results of excluding $\mathrm{SiO}$ from the full set and also just from the AE6 set are shown, respectively, in parts $\mathrm{F}$ and $\mathrm{G}$ of Table 5. Part F of 
(a)

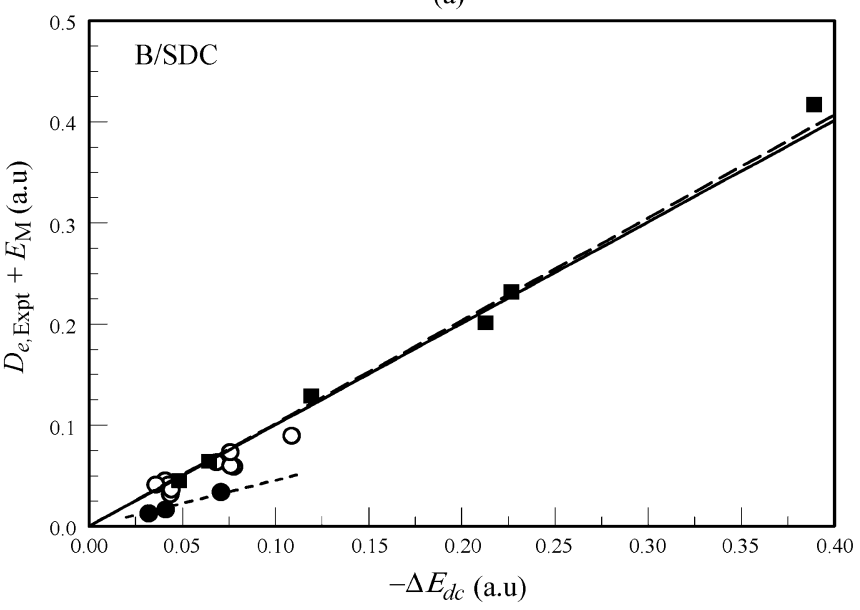

(c)

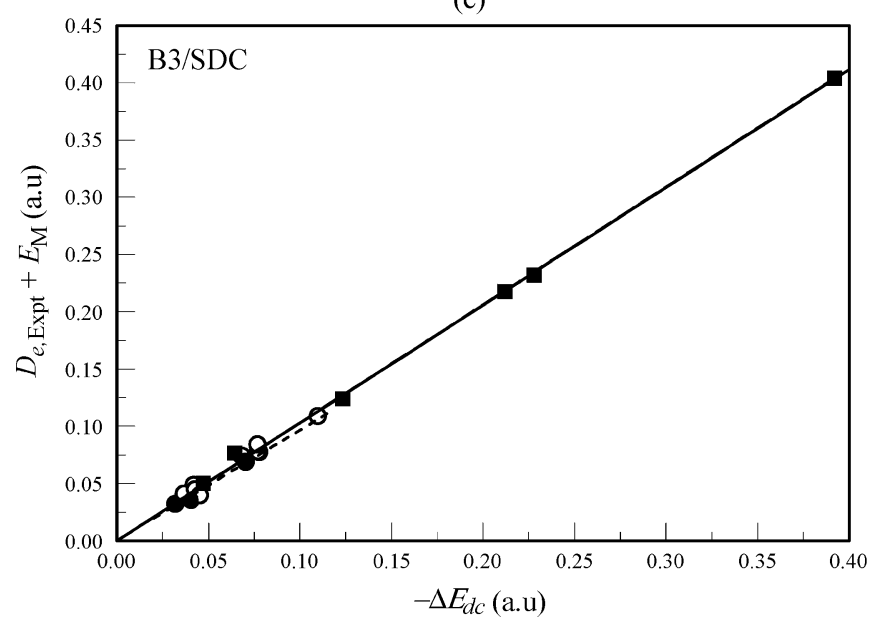

(b)

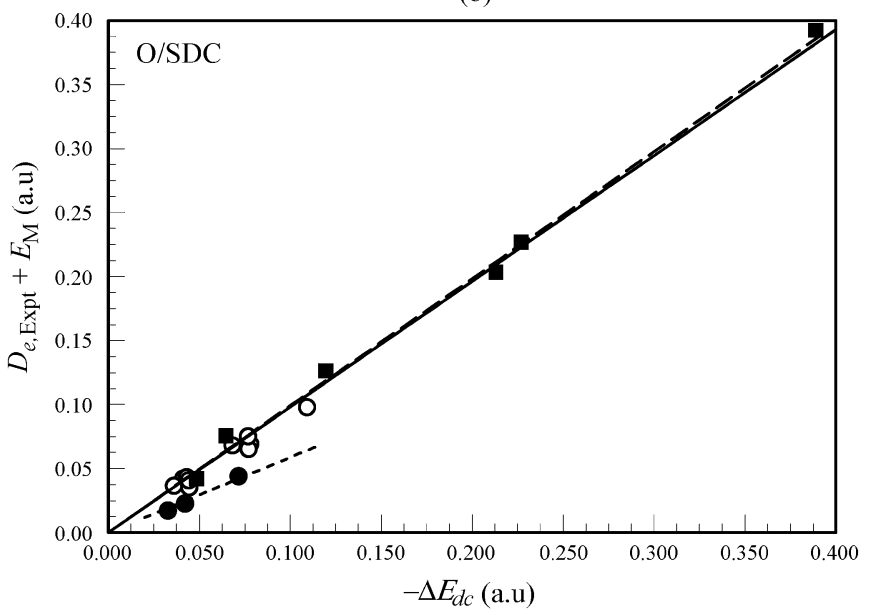

(d)

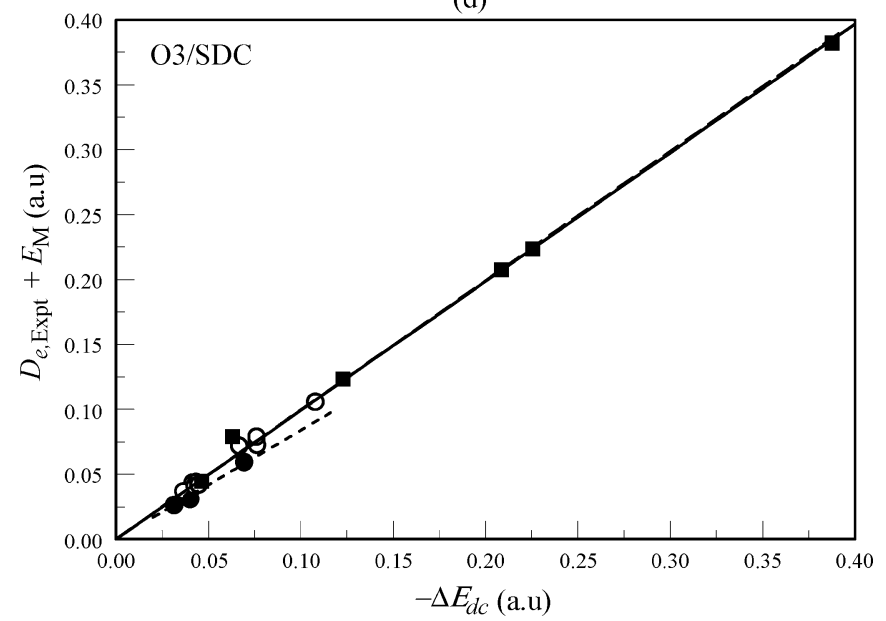

Figure 1. LS analysis for optimal SDC scale factors. Symbols: $\mathbf{\square}$, AE6 molecules; $\mathrm{O}$, non-AE6 molecules except $\mathrm{OF}, \mathrm{F}_{2}$, and $\mathrm{F}_{2} \mathrm{O}$; and $\bullet$, OF, $\mathrm{F}_{2}$, and $\mathrm{F}_{2} \mathrm{O}$. The solid lines are fits to all data; the dashed lines are fits to all except $\mathrm{OF}, \mathrm{F}_{2}$, and $\mathrm{F}_{2} \mathrm{O}$; and the dotted lines are fits to $\mathrm{OF}$, $\mathrm{F}_{2}$, and $\mathrm{F}_{2} \mathrm{O}$ only.

Table 5 shows that it is now possible to achieve a MUE of $1.69 \mathrm{kcal} / \mathrm{mol}$ for the set of 18 molecules with B3 and 1.26 $\mathrm{kcal} / \mathrm{mol}$ with O3. The maximum errors are also substantially smaller for the two hybrid functionals, decreasing almost by a third in the case of O3/SDC, as compared to that in part E. Considering just the five molecules remaining in the AE6 set after excluding $\mathrm{SiO}$, the MUEs are less than half of that reported over the full AE6 set (part B) in the case of B3 and O3, and the maximum errors are smaller by factors of 4 and 5, respectively.

Comparisons to Other Methods. Formally, the DFT methods used here scale as $N^{4}$, where $N$ is the number of atoms in the molecule. In Table 6 , we compare the performance of the SDC approach for the AE6 molecules with those of two MC methods of the Truhlar group, which formally scale as $N^{5}$, for which the errors over the AE6 molecule set are available. ${ }^{32}$ These are the MC3BB and the MC3MPW, ${ }^{32}$ both of which are the result of mixing HF and MP2 with hybrid meta density functionals (meta denotes that kinetic energy density is included in the functional), and each contains three optimized parameters. We compare the mean unsigned error per bond (MUEPB), the mean signed error per bond (MSEPB), and the root-mean-square error per bond (RMSEPB). The per bond error quantities are obtained by dividing the mean error quantities for a method by the mean number of bonds per molecule in the database. Double and triple bonds are all counted as single bonds for the calculation of the mean number of bonds per molecule, which
TABLE 5: LS SDC Scale Factors and the Errors in the Calculated SDC Scaled Atomization Energies

\begin{tabular}{|c|c|c|c|c|}
\hline & $\mathrm{B} / \mathrm{SDC}$ & $\mathrm{O} / \mathrm{SDC}$ & $\mathrm{B} 3 / \mathrm{SDC}$ & $\mathrm{O} 3 / \mathrm{SDC}$ \\
\hline \multicolumn{5}{|c|}{ (A) all 19 molecules } \\
\hline$f^{\mathrm{M}}$ & 1.003618 & 0.981806 & 1.028632 & 0.992286 \\
\hline MUE (kcal/mol) & 7.05 & 4.62 & 2.04 & 2.30 \\
\hline $\max \mathrm{UE}(\mathrm{kcal} / \mathrm{mol})$ & 23.09 & 16.35 & 6.75 & 10.62 \\
\hline \multicolumn{5}{|c|}{ (B) AE6 molecules only } \\
\hline$f^{\mathrm{M}}$ & 1.040921 & 1.002759 & 1.029463 & 0.994385 \\
\hline MUE (kcal/mol) & 5.13 & 3.90 & 1.91 & 2.46 \\
\hline $\max \mathrm{UE}(\mathrm{kcal} / \mathrm{mol})$ & 12.45 & 6.81 & 6.71 & 10.53 \\
\hline \multicolumn{5}{|c|}{ (C) all except $\mathrm{OF}, \mathrm{F}_{2}$, and $\mathrm{F}_{2} \mathrm{O}$} \\
\hline$f^{\mathrm{M}}$ & 1.017170 & 0.991809 & 1.030092 & 0.995976 \\
\hline MUE (kcal/mol) & 5.84 & 3.40 & 2.07 & 1.86 \\
\hline $\max \mathrm{UE}(\mathrm{kcal} / \mathrm{mol})$ & 13.62 & 7.25 & 6.69 & 10.47 \\
\hline \multicolumn{5}{|c|}{ (D) $\mathrm{OF}, \mathrm{F}_{2}$, and $\mathrm{F}_{2} \mathrm{O}$ only } \\
\hline$f^{\mathrm{M}}$ & 0.455117 & 0.588603 & 0.967777 & 0.838358 \\
\hline MUE (kcal/mol) & 1.15 & 1.22 & 1.23 & 0.76 \\
\hline $\max \mathrm{UE}(\mathrm{kcal} / \mathrm{mol})$ & 1.18 & 1.27 & 1.97 & 1.45 \\
\hline
\end{tabular}

(E) all 19 molecules, using two scale factors (C and D)

$\begin{array}{lrrrr}\text { MUE }(\mathrm{kcal} / \mathrm{mol}) & 5.10 & 3.06 & 1.94 & 1.68 \\ \max U E(\mathrm{kcal} / \mathrm{mol}) & 13.62 & 7.25 & 6.69 & 10.47\end{array}$

(F) all molecules except $\mathrm{SiO}$, using two scale factors (C and D) $\begin{array}{lcccc}\text { MUE }(\mathrm{kcal} / \mathrm{mol}) & 3.4 & 2.38 & 1.69 & 1.26 \\ \max U E(\mathrm{kcal} / \mathrm{mol}) & 12.66 & 6.09 & 4.86 & 3.25\end{array}$

(G) AE6 molecules excluding $\mathrm{SiO}$, using scale factor from $\mathrm{B}$

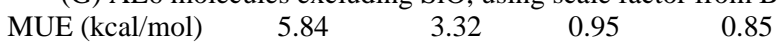
$\begin{array}{lrrrr}\max U E(\mathrm{kcal} / \mathrm{mol}) & 12.45 & 6.29 & 1.57 & 1.92\end{array}$ 
TABLE 6: Comaprison of Mean Errors for the SDC Methods with Those of Two MC DFT Methods for the AE6 Set of Molecules ${ }^{a}$

\begin{tabular}{lrccrrr}
\hline $\mathrm{kcal} / \mathrm{mol}$ & MC3BB & MC3MPW & B/SDC & O/SDC & B3/SDC & O3/SDC \\
\hline MUEPB & 0.47 & 0.71 & 1.06 & 0.81 & 0.40 & 0.51 \\
MSEPB & -0.02 & -0.39 & 0.29 & -0.07 & -0.17 & -0.30 \\
RMSEPB & 0.53 & 0.91 & 0.54 & 0.38 & 0.25 & 0.37
\end{tabular}

${ }^{a}$ The mean signed error is the average of $D_{\mathrm{e}, \text { calcd }}-D_{\mathrm{e}, \text { expt }}$, divided by the mean number of bonds per molecule, 4.83 .

turns out to be 4.83 for the AE6 data set. The signed error is defined $D_{\text {e,calcd }}-D_{\text {e,expt }}$.

Table 6 shows that the MC methods yield smaller MUEPB than the B/SDC and O/SDC methods. However, both B3 and O3 hybrid functionals yield MUEPBs that are slightly lower than those of the MC3 methods. The mean signed error per bond (MSEPB) for the SDC methods is lower than that for MC3MPW, but only O/SDC compares favorably with MC3BB in this category. The two MC3 methods and three of the SDC methods have negative values for this quantity, which means that they underestimate the atomization energy per bond, on average. Three of the SDC methods yield RMSEPB lower than the two MC3 methods. In ref 34, Zhao and Truhlar report six other MC extrapolated density functional methods that scale as $N^{5}$. The MUEPBs for these methods, in $\mathrm{kcal} / \mathrm{mol}$, calculated over a 109 molecule MGAE109/04 atomization energy training set are as follows: MCCO-TS, 0.41; MCCO-MPWB, 0.49; MCCO-MPW, 0.57; MC3MPWB, 0.67; MC3TS, 0.69; and MC$\mathrm{CO}, 0.67 .{ }^{34}$ It seems reasonable to expect SDC scaling to match or exceed these accuracies, especially when applied to the newer exchange-correlation functionals such as the MPWB1K or MPW1B95 of Zhao and Truhlar, ${ }^{33}$ which have been shown to be superior to the earlier generation DFT functionals.

\section{Summary and Conclusions}

We have shown that the correlation energy obtained from the LYP functional of DFT can be scaled so as to increase the accuracy of calculated atomization energies without simultaneously worsening the agreement between the calculated and the experimental structural and spectroscopic properties of molecules. We also showed that optimal scale factors selected by LS analysis can be used to increase the overall accuracy of atomization energies for the entire set, especially if different scale factors are used for subsets of molecules that exhibit different trends.

The results reported in this paper indicate that a simple one parameter scaling of LYP correlation energy calculated using the density from a good hybrid exchange functional such as the B3 or the O3 can yield "chemical accuracy" with less computational effort than many of the MC methods. Because the SDC approach only involves DFT calculations at two levels (with and without the correlation functional), it scales formally as $N^{4}$ (where $N$ is the number of atoms) whereas MC methods based on mixing DFT and correlated ab initio methods scale as $N^{5}$ or $N^{6}$ and multilevel ab initio methods scale as $N^{7}$. The present approach is, therefore, readily applicable to larger molecules.

To implement the present approach for a large molecule, one would perform a geometry optimization and a frequency calculation using an exchange-correlation functional, a single point calculation without the correlation functional, and calculations of atomic energies with and without the correlation functional (so as to obtain relative energies), using the same one electron basis set. Once the molecular dynamic correlation energy is obtained from these calculations, an optimal scale factor would presumably be available to reliably bring the calculated result into better agreement with experiments. SDCscaled harmonic frequencies of the molecule can also be evaluated without constructing potential energy hypersurfaces since, from eq 5

$E_{\mathrm{M}}^{\prime \prime} \mathrm{SDC}=E_{\mathrm{M}}^{\prime \prime}+f^{\mathrm{M}}\left(E_{\mathrm{MLYP}}^{\prime \prime}-E_{\mathrm{M}}^{\prime \prime}\right)=E_{\mathrm{M}}^{\prime \prime}\left(1-f^{\mathrm{M}}\right)+E_{\mathrm{MLYP}}^{\prime \prime}$

where the primes denote differentiation with respect to coordinates. Therefore, harmonic frequencies calculated at the pure exchange and exchange-correlation levels of DFT are sufficient to obtain a reasonable approximation to the scaled frequencies of the molecule. If $1-f^{\mathrm{M}} \approx 0$, the SDC-scaled frequencies will be close to the MLYP results; therefore, in most cases, a frequency calculation at the $\mathrm{M}$ level of treatment will probably be unnecessary. It is thus possible to include zero point energies in the atomization energies (with appropriate scaling of the calculated harmonic frequencies in order to approximate the fundamentals, ${ }^{32,33,61}$ if desired), which permits direct comparison to experimental data corrected only for the spin-orbit effects.

Figure 1 clearly shows that the correlation energy of the six AE6 molecules spans a wide range, which is probably why they are representative of the much larger 109 molecule database of atomization energies. However, before we could make recommendations for optimal scale factors $f^{\mathrm{M}}$, further testing of this approach is clearly needed using different basis sets, second generation exchange-correlation functionals, ${ }^{60}$ and a training set that includes ionization potentials and electron affinities. Scaling that takes into account the types of molecules involved (as in the cases of $\mathrm{OF}, \mathrm{F}_{2}$, and $\mathrm{HOF}$, for example) and bond types may also lead to further improvements.

Acknowledgment. We thank Professor Nicholas C. Handy for helpful discussions on correlation energy.

\section{References and Notes}

(1) Parr, R. G.; Yang, W. Density Functional Theory of Atoms and Molecules; Oxford University Press: New York, 1989.

(2) Löwdin, P. O. Adv. Chem. Phys. 1959, 2, 207.

(3) Sinanoğlu, O. Adv. Chem. Phys. 1964, 6, 315.

(4) Roos, B. O. Int. J. Quantum Chem. Symp. 1980, 14, 175

(5) Werner, H.-J. Adv. Chem. Phys. 1987, 69 (Part I), 1.

(6) Brown, F. B.; Truhlar, D. G. Chem. Phys. Lett. 1985, 117, 307.

(7) (a) Steckler, R.; Schwenke, D. W.; Brown, F. B.; Truhlar, D. G. Chem. Phys. Lett. 1985, 121, 475. (b) Schwenke, D. W.; Steckler, R.; Brown, F. B.; Truhlar, D. G. J. Chem. Phys. 1986, 84, 5706. (c) Schwenke, D. W.; Steckler, R.; Brown, F. B.; Truhlar, D. G. J. Chem. Phys. 1987, 86, 2443.

(8) Lynch, G. C.; Steckler, R.; Schwenke, D. W.; Varandas, A. J. C.; Truhlar, D. G.; Garrett, B. C. J. Chem. Phys. 1991, 94, 7136.

(9) (a) Mielke, S. L.; Lynch, G. C.; Truhlar, D. G.; Schwenke, D. W. Chem. Phys. Lett. 1993, 213, 10. (b) Mielke, S. L.; Lynch, G. C.; Truhlar, D. G.; Schwenke, D. W. Chem. Phys. Lett. 1994, 217, 173 (erratum).

(10) Schwenke, D. W.; Tucker, S. C.; Steckler, R.; Brown, F. B.; Lynch, G. C.; Truhlar, D. G. J. Chem. Phys. 1989, 90, 3110.

(11) Ramachandran, B.; Senekowitsch, J.; Wyatt, R. E. Chem. Phys. Lett. 1997, 270, 387.

(12) Allison, T. C.; Ramachandran, B.; Senekowitsch, J.; Truhlar, D. G.; Wyatt, R. E. THEOCHEM 1998, 454, 307.

(13) Ramachandran, B.; Schrader, E. A., III; Senekowitsch, J.; Wyatt, R. E. J. Chem. Phys. 1999.

(14) Zhang, H.; Ramachandran, B.; Senekowitsch, J.; Wyatt, R. E. THEOCHEM 1999, 487, 75.

(15) Mok, D. K. W.; Neumann, R.; Handy, N. C. J. Phys. Chem. 1996, $100,6225$.

(16) Gritsenko, O. V.; Schipper, P. R. T.; Baerends, E. J. J. Chem. Phys. 1997, 107, 5007.

(17) Lee, C.; Yang, W.; Parr, R. G. Phys. Rev. B 1988, 37, 785.

(18) Perdew, J. P. Phys. Rev. B 1986, 33, 8822.

(19) Vosko, S. J.; Wilk, L.; Nusair, M. Can. J. Phys. 1980, 58, 1200.

(20) (a) Gordon, M. S.; Truhlar, D. G. J. Am. Chem. Soc. 1986, 108, 5412. (b) Gordon, M. S.; Truhlar, D. G. Int. J. Quantum Chem. 1987, 31, 81. 
(21) Gordon, M. S.; Nguyen, K. A.; Truhlar, D. G. J. Phys. Chem. 1989 93, 7356. (b) Rossi, I.; Truhlar, D. G. Chem. Phys. Lett. 1995, 234, 64.

(22) Fast, P. L.; Corchado, J.; Sanchez, M. L.; Truhlar, D. G. J. Phys. Chem. A 1999, 103, 3139.

(23) Seigbahn, P. E. M. Adv. Chem. Phys. 1996, 92, 333.

(24) Fast, P. L.; Corchado, J. C.; Sanchez, M. L.; Truhlar, D. G. J. Phys. Chem. A 1999, 103, 5129.

(25) Fast, P. L.; Sanchez, M. L.; Corchado, J. C.; Truhlar, D. G. J. Chem. Phys. 1999, 110, 11679

(26) Fast, P. L.; Sanchez, M. L.; Truhlar, D. G. Chem. Phys. Lett. 1999 $306,407$.

(27) Tratz, C. M.; Fast, P. L.; Truhlar, D. G. PCCP 1999, 2, 14

(28) Fast, P. L.; Truhlar, D. G. J. Phys. Chem. A 2000, 104, 6111.

(29) Lynch, B. J.; Truhlar, D. G. J. Phys. Chem. A 2002, 106, 842.

(30) Lynch, B. J.; Truhlar, D. G. J. Phys. Chem. A 2003, 107, 3898.

(31) Curtiss, L. A.; Raghavachari, K.; Redfern, P. C.; Pople, J. A. J. Chem. Phys. 2000, 112, 1125.

(32) Zhao, Y.; Lynch, B. J.; Truhlar, D. G. J. Phys. Chem. A 2004, $108,4786$.

(33) Zhao, Y.; Truhlar, D. G. J. Phys. Chem. A 2004, 108, 6908.

(34) Zhao, Y.; Lynch, B. J.; Truhlar, D. G. PCCP 2005, 7, 43.

(35) Lynch, B. J.; Truhlar, D. G. J. Phys. Chem. A 2003, 107, 8996.

(36) Lynch, B. J.; Truhlar, D. G. J. Phys. Chem. A 2003, 107, 3898.

(37) Ramachandran, B.; Peterson, K. A. J. Chem. Phys. 2003, 119, 9590.

(38) Ramachandran, B.; Vegesna, N. S.; Peterson, K. A. J. Phys. Chem. A 2003, 107, 7983.

(39) For an excellent review, see Dunning, T. H., Jr. J. Phys. Chem. A 2000, 104, 9062.

(40) Becke, A. D. Phys. Rev. A 1988, 38, 3098.

(41) Becke, A. D. J. Chem. Phys. 1993, 98, 5648.

(42) Handy, N. C.; Cohen, A. J. Mol. Phys. 2001, 99, 403.

(43) Handy, N. C.; Cohen, A. J. Mol. Phys. 2001, 99, 607.

(44) Frisch, M. J.; Trucks, G. W.; Schlegel, H. B.; Scuseria, G. E.; Robb M. A.; Cheeseman, J. R.; Montgomery, J. A., Jr.; Vreven, T.; Kudin, K. N.; Burant, J. C.; Millam, J. M.; Iyengar, S. S.; Tomasi, J.; Barone, V.; Mennucci, B.; Cossi, M.; Scalmani, G.; Rega, N.; Petersson, G. A. Nakatsuji, H.; Hada, M.; Ehara, M.; Toyota, K.; Fukuda, R.; Hasegawa, J.; Ishida, M.; Nakajima, T.; Honda, Y.; Kitao, O.; Nakai, H.; Klene, M.; Li, X.; Knox, J. E.; Hratchian, H. P.; Cross, J. B.; Bakken, V.; Adamo, C.; Jaramillo, J.; Gomperts, R.; Stratmann, R. E.; Yazyev, O.; Austin, A. J.; Cammi, R.; Pomelli, C.; Ochterski, J. W.; Ayala, P. Y.; Morokuma, K.; Voth, G. A.; Salvador, P.; Dannenberg, J. J.; Zakrzewski, V. G.; Dapprich, S.; Daniels, A. D.; Strain, M. C.; Farkas, O.; Malick, D. K.; Rabuck, A. D.; Raghavachari, K.; Foresman, J. B.; Ortiz, J. V.; Cui, Q.; Baboul, A.
G.; Clifford, S.; Cioslowski, J.; Stefanov, B. B.; Liu, G.; Liashenko, A.; Piskorz, P.; Komaromi, I.; Martin, R. L.; Fox, D. J.; Keith, T.; Al-Laham, M. A.; Peng, C. Y.; Nanayakkara, A.; Challacombe, M.; Gill, P. M. W.; Johnson, B.; Chen, W.; Wong, M. W.; Gonzalez, C.; Pople, J. A. Gaussian 03, Revision C.02; Gaussian, Inc.: Wallingford, CT, 2004.

(45) For example, the route statement "\# OLYP/basis IOp $(3 / 78=-1)$ " results in an OPTX calculation.

(46) Vosko, S. H.; Wilk, L.; Nusair, M. Can. J. Phys. 1980, 58, 1200.

(47) http://comp.chem.umn.edu/database/.

(48) Surfit is described in Spektroskopische Eigenschaften aus electronischen Wellenfunktionen. Senekowitsch, J. Ph.D. Thesis, Johann Wolfgang Goethe Universitaet, Frankfurt am Main, Germany, 1988.

(49) Carter, S.; Handy, N. C. J. Chem. Phys. 1987, 87, 4294.

(50) Huber, K. P.; Herzberg, G. Constants of Diatomic Molecules; Van Nostrand Reinhold: New York, 1979.

(51) Ruscic, B.; Wagner, A. F.; Harding, L. B.; Asher, R. L.; Feller, D.; Dixon, D. A.; Peterson, K. A.; Song, Y.; Qian, X.; Ng, C.-Y.; Liu, J.; Chen, W.; Schwenke, D. W. J. Phys. Chem. A 2002, 106, 2727.

(52) Chase, M. W., Ed. NIST-JANAF thermochemical tables. Phys. Chem. Ref. Data Monograph, 4th ed.; American Institute of Physics: Woodsbudy, NY, 1998.

(53) http://webbook.nist.gov/chemistry.

(54) Miller, C. E.; Drouin, B. J. J. Mol. Spectrosc. 2001, 205, 312.

(55) NIST Computational Chemistry Comparison and Benchmark Database, Release 10, August 2004 (NIST Standard Reference Database 101) (http://srdata.nist.gov/cccbdb/).

(56) NIST Atomic Spectra Database (http://physics.nist.gov/cgi-bin/ AtData/main_asd).

(57) The adjusted $D_{\mathrm{e}, \text { expt }}$ is obtained as $D_{\mathrm{e}, \text { expt }}^{\text {actual }}-\Delta E_{\mathrm{SO} \text {,molecule }}+$ $\Delta E_{\mathrm{SO}, \text { atom, }}$ where the $\Delta E_{\mathrm{SO}}$ are degeneracy-averaged experimental multiplet energies.

(58) (a) Boese, A. D.; Handy, N. C. J. Chem. Phys. 2002, 116, 9559. (b) Boese, A. D.; Martin, J. M. L.; Handy, N. C. J. Chem. Phys. 2003, 119 3005 .

(59) Baker, J.; Pulay, P. J. Chem. Phys. 2002, 117, 1441.

(60) (a) Lynch, B. J.; Zhao, Y.; Truhlar, D. G. J. Phys. Chem. A 2003, 107, 1384. (b) Zhao, Y.; Pu, J.; Lynch, B. J.; Truhlar, D. G. PCCP 2004, 6,673 .

(61) Sinha, P.; Boesch, S. E.; Gu, C.; Wheeler, R. A.; Wilson, A. K. J. Phys. Chem. A 2004, 108, 9213.

(62) Wang, N. X.; Wilson, A. K. J. Chem. Phys. 2004, 121, 7632.

(63) Johnson, E. R.; Wolkow, R. A.; DiLabio, G. A. Chem. Phys. Lett. 2004, 394, 334. 